\section{BARRERA ANTIMAREAS EN EL TAMESIS GRAN BRETAÑA}

\author{
Rendel, Palmer y Tritton, \\ Ingenieros
}

$533-24$

\section{SINOPSIS}

La «Barrera del Támesis» es uno de los sistemas contra inundación fluvial más ambicioso que se ha realizado hasta ahora en el mundo.

Está formada por una serie de compuertas móviles soportadas entre pilares de hormigón, que descansan sobre el lecho del río y permiten el paso del tráfico fluvial. Dispone de: cuatro compuertas de $61 \mathrm{~m}$ de luz cada una; dos compuertas con una luz de 31,5 m, y otras cuatro, de menores dimensiones, situadas en los extremos donde el agua es de escasa profundidad y no existe tráfico comercial.

La necesidad de la construcción de esta Barrera viene determinada porque Londres se hunde gradualmente en su suelo de arcilla, por lo cual el nivel del agua en el Puente de Londres se ha elevado unos $61 \mathrm{~cm}$ en el curso de los últimos 100 años.

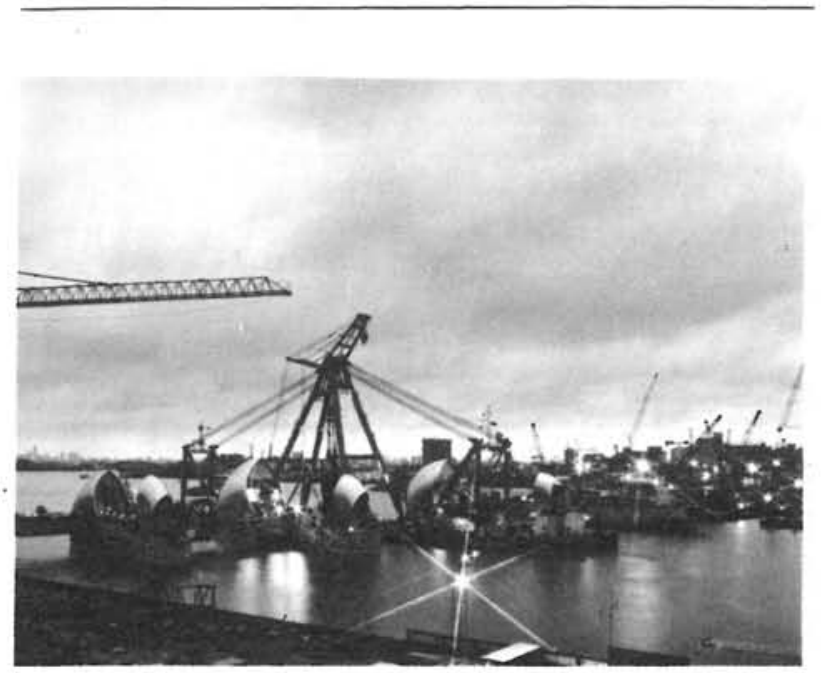

Cuando los expertos tratan del tema de la inundación del Támesis no sólo la consideran posible sino que hablan de ella como una certeza.

Esa convicción se basa en tres factores: dos permanentes y un tercero ocasional. El primer factor es consecuencia de que Londres se apoya en cimentaciones de arcillas. El segundo se debe a que el conjunto de Gran Bretaña se inclina en la dirección sudeste a una velocidad de cerca de 60 centimetros cada 100 años. El tercer factor se basa en la marea, ayudada por vientos fuertes del norte y lluvia pesada, que puede avanzar aguas arriba del Támesis.

Esta marea se produce cuando una baja presión en el atlántico eleva el nivel del agua, que atraviesa el Norte de Escocia y puede alcanzar la zona poco profunda del Mar del Norte y entrar en el cuello de botella que forman el sudeste de Inglaterra, Bélgica y Holanda.

La inundación producida, si no existiese la barrera del Támesis, alcanzaria alrededor de $116 \mathrm{~km}^{2}$ en el Londres Central, con una población de un millón de personas.

Como consecuencia de esta posible inundación, las Sedes del Parlamento y Whitehall -donde están situados la mayor parte de los departamentos del gobierno central- podrian quedar $1 \mathrm{~m}$ por debajo del nivel del agua durante 1 dia, mientras que la Isla de Dog en el río quedaría $6 \mathrm{~m}$ bajo el nivel de las aguas durante 6 dias. La parte central del Metro de Londres podria quedar inutilizada para unos 6 meses y la mayoria de los servicios, tales como energia eléctrica, gas, agua, saneamiento y telecomunicaciones podrian, asimismo, interrumpirse.

Aparte de las pérdidas de seres humanos, el coste total de la inundación estaria entre unos 300 y 400.000 millones de pesetas.

\section{Realidad histórica}

Estas inundaciones del Támesis no sólo se consideran posibles en el futuro, sino que constituyen una realidad histórica.

Se sabe que ya en el año 1236 los hombres pudieron ir en bote de remos al Palacio de Westmeinster, entonces residencia del monarca británico. 


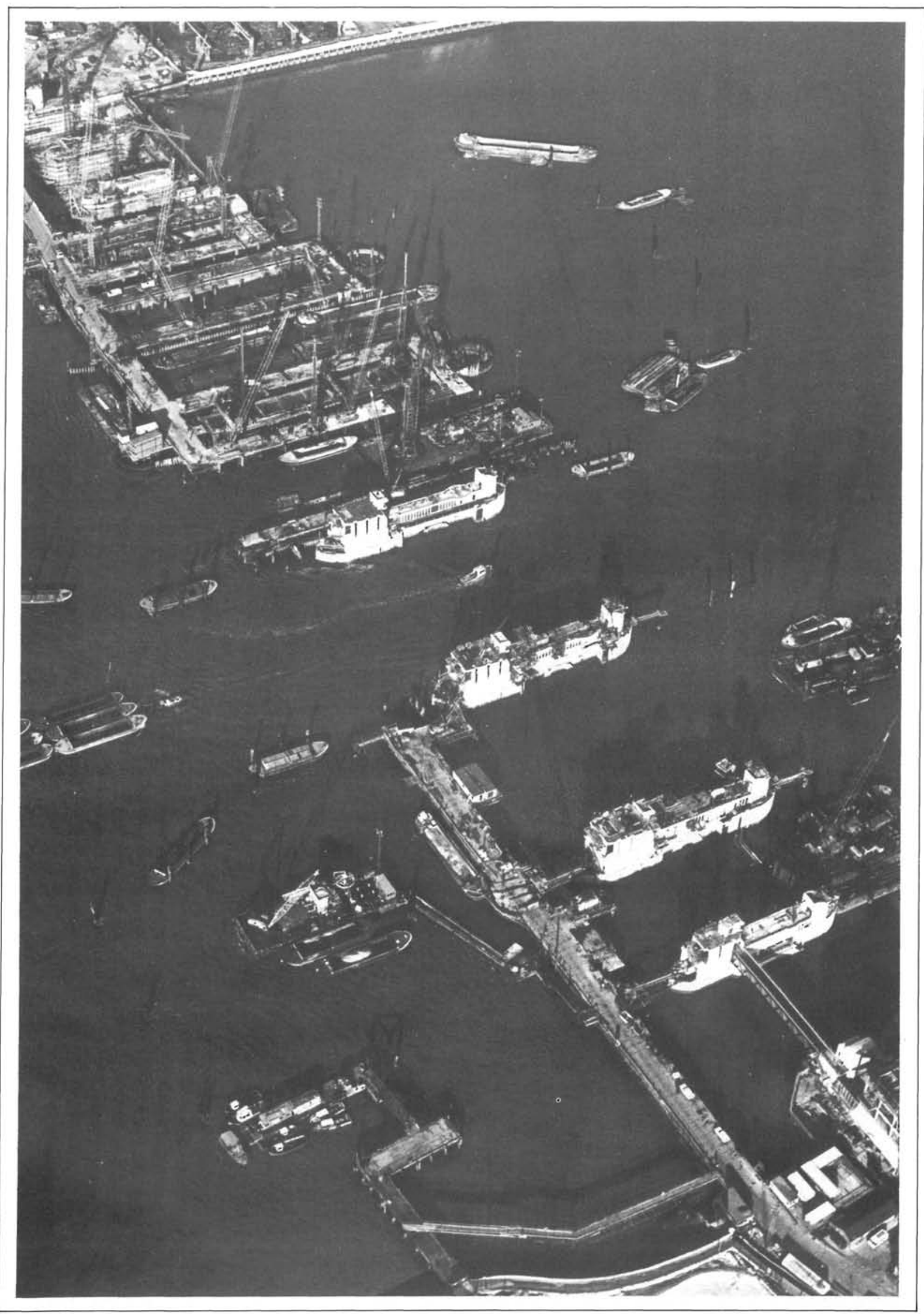


Igualmente algunos famosos periodistas aseguran que en 1663 "todo Whitehall llegó a estar sumergidon.

En 1928 catorce personas murieron en el Londres Central, cuando se produjo una gran crecida en el Támesis lo cual produjo su desbordamiento. Asimismo, en 1953, hubo una serie de inundaciones desastrosas que arrasaron la costa este de Inglaterra y el estuario del Támesis provocando la muerte de 300 personas, un tercio de las cuales vivia en el área de Londres a pesar de que el agua no alcanzó la zona más poblada de la ciudad.

Las más recientes amenazas del Támesis fueron, en 1978, cuando en dos ocasiones un punto del rio inundó $25 \mathrm{~cm}$ sus orillas.

El método más normal para evitar las inundaciones de un rio es elevar y asegurar sus muros y terraplenes, por lo que este método fue el empleado en el Támesis hasta el año 1971.

Sin embargo, esta solución se vió que no era válida debido a que se comprobó que, como consecuencia del incremento de la altura de los muros, se empezaba a perder la vista del Támesis y de la famosa alameda que se extiende a lo largo de sus orillas, desde la que miles de turistas exploran Londres. A consecuencia de esto, en 1972, el Parlamento aprobó la construcción de una barrera para unir North Woolwich en la orilla sur del Támesis con Silvertown en la orilla Norte.

La solución de barrera fue largamente discutida en el siglo XVIII. En todos los proyectos de este siglo se incluia como solución la construcción de una presa permanente con esclusas de embarque, compuertas gigantes de acero guiadas desde la orilla mediante ruedas de caucho y una especie de rastrillo grande con una compuerta abatible.

\section{Proyecto}

Esta solución anterior no fue elegida debido a sus consecuencias en el medio entorno. El proyecto finalmente escogido consiste en la construcción de diez compuertas de acero colocadas entre nueve diques de hormigón y las orillas del río. La maquinaria hidráulica necesaria para mover estas compuertas se sitúa en la parte superior de los diques.

Seis de las compuertas se han proyectado huecas, tipo sector, y sección D. Funcionarán en sentido ascendente y cuando no estén en uso quedarán curvadas con su sección D hacia abajo sobre el lecho del río. En esta posición se apoyarán sobre unas vigas de hormigón pretensado. Estas compuertas se podrán, en una sola operación, levantar y girar 90 grados.
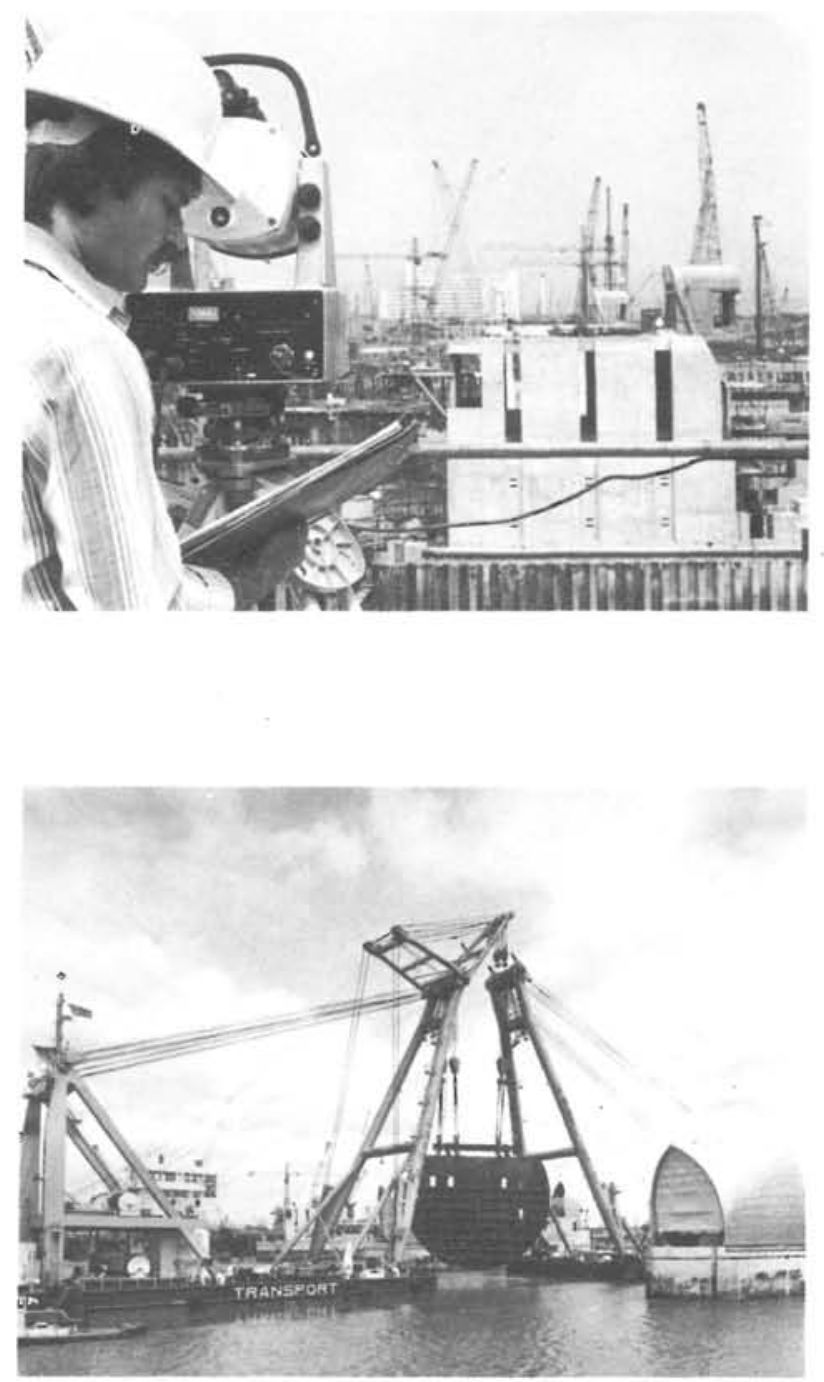

Las bóvedas protectoras de la maquinaria serán de madera de iroko y madera roja, recubiertas de acero inoxidable.

Las otras cuatro compuertas del tipo radial, descendente. Irán suspendidas entre los diques y al igual que las compuertas-sector podrán girar $90^{\circ}$ para situarse en la posición correcta. Por estas compuertas no pasará tráfico comercial.

Es de destacar que se han empleado técnicas muy avanzadas de medición para la construcción de las Barreras del Támesis. Estas técnicas comprenden la utilización del Tellsometer MA100, equipo electro-óptico de medición que proporciona una exactitud de $\pm 0,5 \mathrm{~mm}$.

Para cerrar todo el sistema de barrera se tardará entre media y una hora, consiguiéndose un muro de $560 \mathrm{~m}$ de longitud de resistencia contra la marea.

Las cuatro compuertas-sector mayores tienen una luz de $61 \mathrm{~m}$, situadas a $20 \mathrm{~m}$ de altura, pesan 


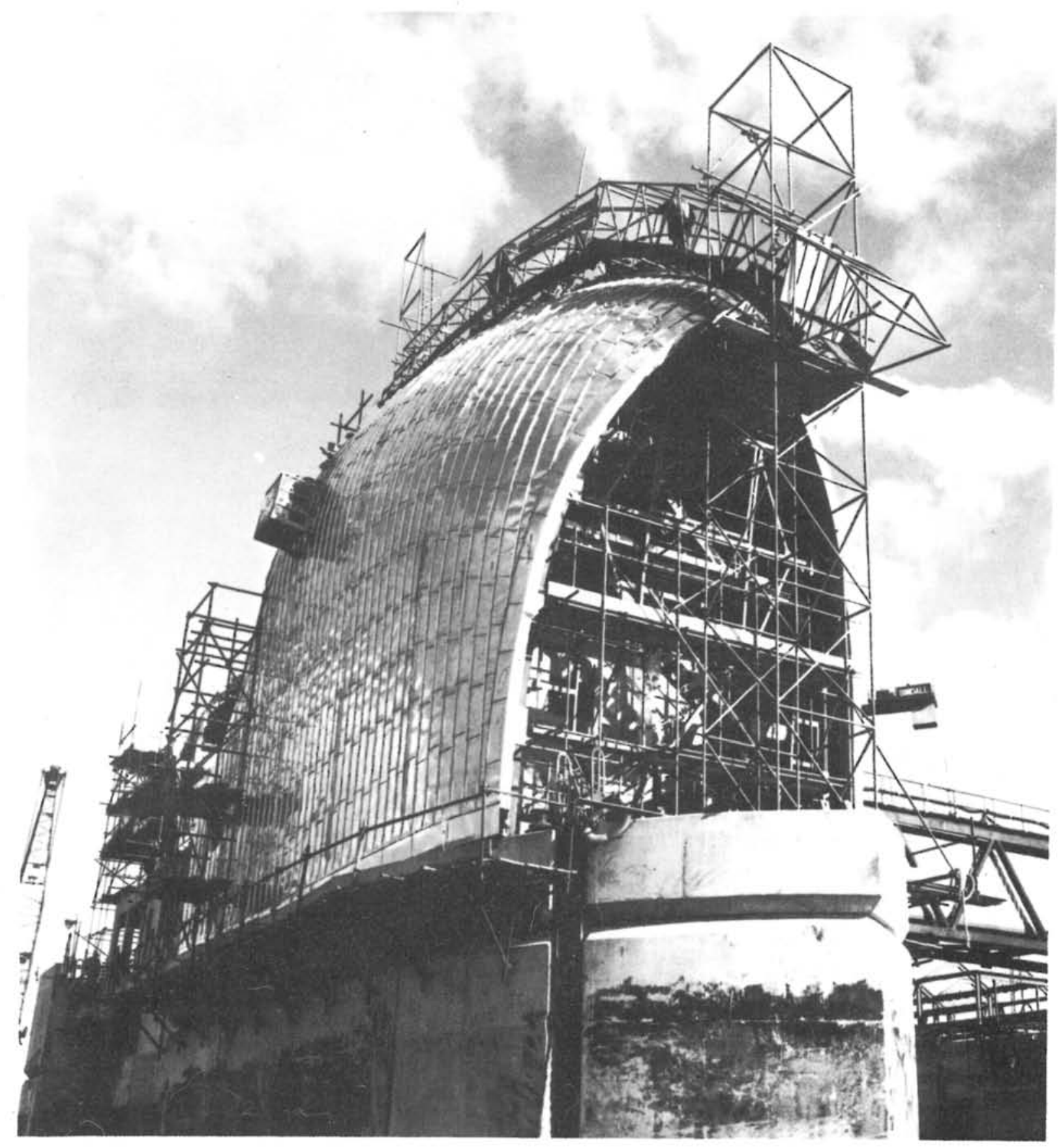

cerca de $3.300 \mathrm{t}$ con contrapesos y son capaces de resistir un peso de más de 9.000 toneladas.

Las compuertas-sector menores y las compuertas radiales tendrán una luz de $31,5 \mathrm{~m}$ y pesan 900 y $200 \mathrm{t}$, respectivamente.

Los cinco diques mayores tienen $50 \mathrm{~m}$ de altura desde el lecho. Estarán situados sobre bases de hormigón realizadas cada una de ellas con un bombeo continuo de hormigón durante 4 dias.

\section{Espacio libre para la nevegación}

El espacio de $61 \mathrm{~m}$ existente entre cada uno de los diques mayores es adecuado para que pasen los barcos, con lo que no se impide el importante flujo de tráfico existente en el Támesis.

Aunque la alarma de la inundación sólo obligará en los años 1980, a cerrar la barrera dos veces al año, sin embargo, en el siglo $\mathrm{XXI}$ el número será de unas 10 veces al año. 